\title{
Opportunities and challenges for enhancing of preconception health care among reproductive age groups of women in Mekele, Tigray Ethiopia 2018/9.
}

abera haftu melkamu ( $\square$ aberahaftu@gmail.com )

Mekelle University College of Health Sciences

brhane G/her G/mikael

Mekelle University

hadgay hagos adanu

Mekelle University

mhiret-ab mehari reda

Mekelle University

kahsay zenebe G/selasie

Mekelle University

shewit adane desta

Mekelle University

Research article

Keywords: opportunities, challenges, enhancing, preconception care

Posted Date: September 24th, 2019

DOl: https://doi.org/10.21203/rs.2.14904/v1

License: (c) (1) This work is licensed under a Creative Commons Attribution 4.0 International License.

Read Full License 


\section{Abstract}

Background Preconception care involves in preventing and detecting of early disease before pregnancy occurred and has potential benefit both for the mother and her fetus. It prevents plenty of perinatal complications and now a days well organized and systematic preconception care services is mandatory for all reproductive age groups.

Objective To explore opportunities and challenges for enhancing preconception care in Mekele, Tigray Ethiopia 2018/9.Method s: Phenomenology study design was employed and sample size was determined by saturation of data. Purposive sampling technique was used to recruit study participants and method of data collection was in-depth interview. Thematic analysis was applied and it was done by atlas ti 7 software. Audios were transcribed first and translated to English version, then after codes and families were given and finally themes were developed.

Result In this study a total of ten reproductive age group females were interviewed and their minimum and maximum age were $24 \& 43$ years respectively. Opportunities and challenges were explored from the participants' perception; they explained that health extension workers, previous experience, family planning (condom) and friend's incident were among contributing factors for enhancing of preconception care and in the counterpart carelessness, less awareness, unplanned pregnancy, unwillingness and poor paternal coordination were the challenges mentioned by the respondents which were recognized as obstacles not to attend preconception care. They explained that again failure to attend preconception care may end up with complicated perinatal outcome like anemia, abortion, hypertension, neural tube defect, preterm labor and low birth weight.

Conclusion and recommendation overall the participants had poor awareness and little experience of preconception care. Preconception is overlooked and less attention is given based on the respondents thought, though it has invaluable contribution in future health of both the mother and fetus. The health system should gave emphasis to prevent those maternal and neonatal complication by mobilizing both the community members and health professionals.

\section{Introduction}

Enhancing of preconception health doesn't merely only focus on reducing risk of poor perinatal outcome but also risk of emerging non-communicable disease in future life (1). If a woman didn't attend preconception care properly, there are obvious and observable complications on pregnancy outcome (2). People with low income and lowest social gradient usually face profound risks to have complicated pregnancy outcome (3). In addition of this, obstetric follow up and receiving of care during preconception period is lower among who are socioeconomically disadvantaged (4).

Perception has great influence whether women should prepare for pregnancy or to use proper preconception care and lacking of adequate perception or awareness may lead to have poor pregnancy preparation (5). Preconception health care involves in detecting of general health status and preventing 
of future diseases both for the mother and fetus as well, diseases like neurologic deformity may probably prevented if a women have proper preconception care (6).

Women's poor awareness and their weak perception towards to preconception care was the routinely identified barriers or challenges of preconception care (7). Most studies focus on known medical diseases and desire to have pregnancy but less attention was given to evaluate all health aspect of couples and their desire to have planned pregnancy and well managed preconception care (8). From WHO reports preconception care mainly focus on sexual and reproductive health (2).

Many researches showed ample evidences that promoting and enhancing of women's health before pregnancy has favorable and positive outcome and highly reduces pregnancy complications, neurological defects, low birth weight and preterm labor (9). Beside these evidences report from center of maternal and child health center research, pre pregnancy care is efficient contributing factor in reducing of maternal mortality (10).

Even if the issue of preconception care was raised and to be topic of emergency in developing countries since long time almost twenty years, the complications are still major health problems; according to WHO estimation 5-7\% live births are preterm labor in developing countries (11). In United States almost half of their pregnancies are unwanted and unplanned, Iran $31.6 \%$, Mashhad $35.8 \%$ of their pregnancies are unplanned (12-14).

Proper utilization of preconception care contains preventive, therapeutic and behavioral interventions, example life style changes, vaccination and genetic screening and using of different medications for chronic medical illness (15). Preconception guidelines and recommendations have been commenced in many developed countries, those recommendations are fragmented and not adequate enough to address the issue of preconception care (16). Lack of knowledge was one of the main barrier in United Kingdom not to deliver preconception health (16).

Perceptions, belief and attitude are much difficult to be measured with quantitative study, so in order to get deep thought and fruitful ideas about preconception care conducting of qualitative study is preferable and this is the main reason considered to conduct this study.

\section{Methods}

\section{Study setting}

\section{Study area and period}

The study was conducted in Mekele Tigray, specifically in Ayder sub city. Tigray is located $783 \mathrm{~km}$ away from the capital city of Ethiopia, Addis Ababa. Mekele is one of the seven zones of region Tigray with population density of 215,546 among this 110,788 of them are women according to census 2009 . The 
fertility rate of region Tigray on average is 3 and for Mekele is 2.7.The study was conducted from September 1, 2018 to August 30, 2019.

Study Design: Phenomenology study design was employed

Source population: All reproductive age group females in Mekele city

Study population: All selected reproductive age group females in Mekele city

\section{Eligibility Criteria}

Inclusion criteria:

All reproductive age group females in Mekele city

Exclusion criteria

Reproductive age females who are mentally unconscious were excluded from the study

\section{Sample size Determination:}

Sample size was determined by level of saturation, ten individuals was interviewed and idea was saturated with this sample size.

\section{Sampling Techniques}

From Mekele city one sub city was selected randomly which was Ayder sub city, then after possible discussion made with the key informants, they give us critical information whom should we interview regarding preconception care. Purposive sampling technique was employed to recruit the study participants after the possible site and target groups get identified.

\section{Data collection method}

First semi structured questioner was developed and the questioner was reviewed by the team members and possible training was given to the data collectors. 3 MSC holders, 1 MSC student and 2 BSC midwives were participated in the data collection. The method of data collection was In-depth interview. After every interview, debriefing and discussion was made to set data saturation and to find where the uninvestigated area is and to focus on the next interview.

\section{Data Quality assurance}


Semi structured question was prepared as a leading point and that questions were discussed by the team members before the actual work and for the consistency and possible modification pilot was done. Every day after collecting of the data there was debriefing and cross check up by all our team members.

\section{Method of Data Analysis}

Audios were transcribed verbatim and it was entered to Atlat ti7 for analysis. Codes and families were developed in the software and finally themes were identified.

\section{Operational Definition}

Opportunities: Every possible chances acquired to enhance the preconception care

Challenges: Any factors which hinders to receive preconception care

\section{Study Variables:}

Dependent variable: Enhancing preconception Health

Independent variables: Opportunities and challenges (perception, unplanned PX, risk assessment)

\section{Ethical Consideration}

Ethical clearance was obtained from institutional review board of Mekele University, College of health sciences and support letter was written from Tigray Regional Health Bureau to the respective administrations of the health facilities. All participants were informed of the objective the study, anonymity of the study was also informed that they are free to withdraw at any time if they want to do so. Finally written informed consent was obtained from the participants.

\section{Dissemination of the Result}

Finally the findings of the study will be submitted to the Tigray Regional Health Bureau and Tigray Public Health institutions. It will also be disseminated in different professional workshops, and conferences. Finally it will be published in internationally recognized journal for a wider visibility.

\section{Results}

\section{Socio Demographic characteristics of study participants in Mekele, Tigray Ethiopia}


A total of ten women's were interviewed and the discussants were from Mekele town Ayder sub city, the minimum and maximum age were 24 and 43 respectively and majority of them were unemployed. Table 1

Table 1: socio demographic characteristics of study participants in Mekele, Tigray Ethiopia

\begin{tabular}{lll} 
Variable & Frequency & Percent \\
\hline Age & & \\
\hline $18-25$ & 3 & $30 \%$ \\
\hline $26-35$ & 5 & $50 \%$ \\
\hline $36-45$ & 2 & $20 \%$ \\
Occupation & & \\
\hline Employed & 2 & $20 \%$ \\
\hline Unemployed & 8 & $80 \%$ \\
\hline Marital status & & \\
\hline Single & 1 & $10 \%$ \\
\hline Married & 8 & $80 \%$ \\
\hline Divorced & 1 & $10 \%$ \\
Religion & & \\
Orthodox & 6 & $60 \%$ \\
\hline Muslim & 3 & $30 \%$ \\
\hline Others & 1 & $10 \%$
\end{tabular}

\section{Knowledge, perception and experience of reproductive age women towards to preconception health}

From the respondents perspective most of the discussants defined preconception care as simple as keeping of personal hygiene and getting of balanced diet, this statement showed that the discussants lack adequate knowledge and importance of preconception care before pregnancy happened. Due to many obstacles preconception care is the most neglected part of reproductive health. One of the discussant explained that this period is a period where to check your general health status.

"To me this preconception care is keeping of your personal hygiene and getting of balanced diet to keep your health status before pregnancy."

"I understand that this is the time to check and treat your general health status since this is the beginning of pregnancy."

Even if the discussants have little experience to preconception care, most of them have positive implication and perception to preconception health care and they believe that this care is fundamental for future generation makeup. One of the discussants explained that mothers have vital role in building of generation and to do this they have to keep their health before pregnancy happen and have to be treated if get ill before any pregnancy happened during preconception health care period. 
"We mothers have great role in continuation of generations and have to maintain our health in order to achieve this aim."

The discussants have no much experience in maintaining of their preconception health, most of them mentioned that before they get pregnant they checked only status of HIV, most of the preconception health care related items were missed in most of the participants. One of the discussants get screened in pre pregnancy period for diabetes, this is because of the participant body weight was more than $90 \mathrm{KG}$ and being afraid of some chronic complications. Among the discussants one of them mentioned that from her real experience the public institutions are less devoted and committed to teach and provide services about preconception health and most of the time clients move to private sectors and they get exposed to unnecessary financial expenses. Probably this could be due to client flow and in some conditions it might be because of carelessness and less awareness of the professionals working in public sectors for preconception health care services.

"Yes I was about $90 \mathrm{~kg}$ and get afraid of some chronic medical illness and I decide to get screened of diabetes before I get pregnant and my husband was in line with this idea and we did it together."'

"From my experience, professionals who are working in public institutions are not voluntary and committed to provide services related to preconception health and personally I preferred to get services from private clinics like Mary stops and bethel."'

All in one the discussants elaborated that woman should get vaccinated for tetanus toxoid before pregnancy occurred and one of them explained that vaccination for measles and polio is important before pregnancy. The participants mentioned that before pregnancy happen couples should get screened for HIV, blood group, diabetes, hypertension and STI. Unexpectedly one of the discussant explained that the normality of the female reproductive organs has to be checked for its anatomic placement like the uterus and cervix. This is great point again that has to be checked and corrected before pregnancy occurred since these malformations are responsible for many obstetrical complications and infertility as well.

"Yes woman has to check blood group, HIV status, STI, diabetes and hypertension before pregnancy period for better outcome."

"Beside all the tests I mentioned so far, females need to check the normality of her reproductive organs in preconception period so as to maintain and enhance their reproductive health."

\section{Source of information about preconception health among reproductive age women's in Mekele, Tigray Ethiopia.}

The discussants had explained that source of information for preconception care benefits is gained from different resources and events. Most of them mentioned their primary source is from media, health 
professionals, own experience, while using contraceptive and reading. One of them described that her source was from internet, using face book, so people can use internet for health issue beyond the social services provided in face book. In addition of this they deeply stated that the role of partners and health institutions is the most important thing in disseminating of the information and enhancement of the preconception care.

One of the participants explained that "Edir" leaders are best to convey and convince the community members if they get full awareness about preconception health care. These leaders are selected in given community based on their experience and social interaction to resolve any conflicts and mange social crisis, so these individuals are well respected and at the same time their sound is worthy in maintaining of any social activities including health related issues.

"My source of information regarding preconception care is from media, health professionals, and reading and from my friends."

"Unlike others I do have my friend working in private sector and always when we talk and chat each other we share information using the messengers and face book regarding sexual related issues."

"In my thought Edir leaders are best to transform this agendum to the community since they are well accepted member of the society."

\section{Opportunities for enhancement of preconception health among reproductive age women in Mekele, Tigray Ethiopia.}

There are multiple opportunities which might enhance preconception health care services, where those services should serve as bench mark of pregnancy. The discussants mentioned that a good opportunity for them to know and enhance their preconception health care were health extension workers, these professionals are hand and glove with Ethiopian society.

This movable health extension workers went to the community to teach health related issues and this program is supported by policy of the nation, since these professionals are in close contact with mothers, they could teach and counsel them the pros and cons of preconception health care services for the society. Some of them said that having health institutions around residency and previous experience are nice means for enhancing of preconception health, according to this thought health institutions are responsible to community to disseminate information and knowledge to the nearby residents about the benefits of the preconception health care services.

One of the participant explained that using condom is best opportunity to capacitate preconception health. This idea is mainly associated with using of condom before pregnancy would prevent her from unintended pregnancy and sexual transmitted diseases and addressing of these complications is the primary target of preconception health care services. 
"Health extension workers are primary sources for enhancing of one's general health specifically preconception care services, again nearby by institutions and experiences are good opportunity to enhance preconception health."

"Indeed there are many positive factors that enhance preconception health but I consider using condom is best of all to capacitate and strengthen pre pregnancy health."

In majority of the discussants support from husband, family and peers are strong factors to enhance preconception health and together with this explanation two of the discussants mentioned that keeping of one's personal hygiene, using of family planning are very important parameters to fortify preconception health care services.

Generally meeting of preconception care is prerequisite to start pregnancy and to complete health pregnancy. The discussants elaborated that preconception health care service opportunities are very important for woman to have successful pregnancy period, health fetus and HIV free newborn, meaning to say if woman get screened and tested for chronic and infectious diseases, she would end up with uncomplicated pregnancy.

"The opportunities would help to have HIV free newborn ....emm to get healthy fetus and over all to complete your pregnancy successfully, those opportunities have great impact in pregnancy outcome."

Challenges/barriers/ of preconception health among reproductive age women's in Mekele, Tigray Ethiopia.

Woman's face a lot of challenges and obstacles to meet their interest in preconception health, those challenges really starts from home and may affect future life of the mothers and her offspring. The participants mentioned that carelessness, lack of awareness and less husband's cooperation were the main barriers not to get adequate preconception health care. From the point view of the discussants the husband level of education and income /economy/ matters to attend proper preconception health care services. One of the discussant explained that during hospital visit to get any services or related services to preconception care, professionals are not much interested to counsel and explain the usage and benefit of preconception health. This is an important explanation if the professionals are not positive and open for discussion regarding the health care issue before pregnancy the people will get loss hope and will probably undermine the benefits of preconception care for the mothers and offspring's as well.

"To me the main challenges or barriers not to attend preconception health care are carelessness, lack of awareness and lack of husband's cooperation. Let say from my personal experience my husband was not interested in having of preconception care, simply I start my follow up after I get pregnant."

"What I understand factors hinders the preconception health is unwillingness of health professionals to counsel and teach about this care during hospital visit for other related services with preconception health." 
The discussants explained that the effect of those barriers on the fate of the mother and fetus is tremendous, based on the discussants' view if a woman doesn't attend her preconception care, she might be predisposed to many diseases like hypertension, diabetes, anemia, early pregnancy bleeding, abortion, unintended pregnancy and heart failure.

Among the discussants one of them elaborated that if preconception care not delivered properly, there will be congenital deformity and neurological disorders in the fetus. Pre pregnancy care is fundamental and basic for the wellbeing of the fetus and mothers as well, especially in the first one month a head of pregnancy supplementation of folic acid is vital for prevention of congenital defect.

one of the interviewee said that if a woman didn't attend preconception care, she might end up with low birth weight and preterm labor, according to the view of the respondent this low birth weight and preterm labor is directly related to preconception care as if the status of the pregnant woman was not determined before pregnancy (i,e screening for medical problems like hypertension and diabetes), she will end up with many maternal and fetal complications.

"Hmm yea there are many effects in future pregnancy if preconception health is hindered ...like hypertension, diabetes, abortion, unintended pregnancy, heart failure anemia, early bleeding, congenital deformity and neurological problems"

"Yes it has an effect, like low birth weight and preterm labor this are some of the common problems me and my friends face during our experience"

\section{Discussion}

In this study the participants had been interviewed not only for opportunities but also for challenges of preconception care, the respondents were less aware of preconception care but with positive perception towards to preconception health care services. Again the participants had poor experience in their index pregnancy to receive preconception care. Among the opportunities the participants considered to enhance preconception care were the health professionals particularly health extension workers, nearby health institutions, previous experience and using of family planning specially condom were the top listed contributing factors for a woman to commence preconception care.

In the other side the respondents explained that what challenges/barriers / affect preconception care were carelessness, less awareness, unplanned pregnancy, uncooperative husband and unwillingness of the professionals had negative impact in having or considering of preconception care.

Unsatisfactory awareness towards to preconception care mentioned in this study is in line to a study conducted in UK(17), where women's from multi ethnic were interviewed and responded that they have limited knowledge to preconception care. "unintended pregnancy " and less attention to pre pregnancy care were again among the barriers here mentioned in this study which is similar to a study conducted in 
UK,USA and Australia where these factors were recognized as challenges for preconception health $(15,18,19)$.

The study conducted in UK, among health care providers GP and Nurses were among the opportunities to provide preconception care on time and appropriately (17), unlike to this result in this study the health extension workers were the primary opportunities to flourish respondents to have preconception care. This variation might be again due to the health policy of our country is different from UK, where prevention strategy is delivered with this health professionals and women developmental army.

Unwillingness and less husband's cooperation were added results to the existing literatures in which these factors were considered as challenges or barriers to begin or continue preconception care in the specified time and condition.

\section{Strengths And Limitations}

One of the strongest side of this study is, it's conducted in well-deserved age group women and it's the first in kind in the region to address preconception care. The limitation is less representative, meaning to say because of budget limitation the study was conducted in single sub city of Mekele town.

\section{Conclusions And Recommendations}

The respondents have limited knowledge and little experience towards to preconception care but promising perception /attitude / of this service. The top mentioned opportunities to enhance preconception care was health extension workers, previous experience and friends incident, whereas challenges for these services were unplanned pregnancy, economy, carelessness, less awareness and professionals unwillingness which hinders preconception care. The respondent again explained that unless a woman had received preconception care appropriately, she may end up with complicated perinatal outcome like abortion, neural tube defect, preterm labor and low birth weight. In order to alleviate these complications the health system has to give great emphasis to preconception care and awareness has to be created among reproductive age women's to start pre pregnancy preparation so as it helps to improve future pregnancy

\section{Abbreviations}

HIV: human immune deficiency virus

PC: preconception care

STI: sexual transmitted infection

WHO: world health organization 


\section{Declarations}

\section{Authors' contributions}

AH had participated starting from drafting of the proposal, data collection, data analysis and manuscript writing. $\mathrm{HH}$ drafted the proposal and revised it critically in the design and analysis of the research. MM was involved mainly in data collection, design and revising in draft of the manuscript and interpretation of the final result. BG was involved in atlas ti7 analysis and theme development. KZ participated in analysis of the research. SA involved in data collection and manuscript writing. All authors read and approved the final manuscript.

\section{Acknowledgements}

We would like to appreciate to Mekele university and midwifery department for giving great opportunity to conduct this study. Our gratitude again goes to our friends who helped us in different way to achieve our work in this way.

\section{Competing interests}

We have no any competing interest.

\section{Availability of data and materials}

The data sets used during the current study are available from the corresponding author on reasonable request.

\section{Consent for publication}

Not applicable.

\section{Ethics approval and consent to participate}

Ethical clearance was obtained from Mekele University, College of Health Sciences Ethical review board. Permission letters were also sought from Tigray Regional education Bureau and the letter was distributed to each selected sites. Written Informed consent from the participants was obtained after clear explanation of the purpose of the study.

\section{Funding}


Mekele University was our fund organization to conduct this study. The role of Mekele University was providing appropriate training to develop the proposal, funding money to our data collectors and following how the study is going on, finally our University provides us basic training which was helpful for our study.

\section{References}

1. CDC. Recommendations to improve preconception health and health care-United States: a report of the CDC/ATSDR Preconception Care Work Group and the Select Panel on Preconception Care. MMWR Recomm Rep 2006; 55 (No. RR-6):1-23.

2. Health Council of the Netherlands. Preconception care: a good beginning. The Hague: Health Council of the Netherlands, 2007.

3. Office for National Statistics. Infant and perinatal mortality in England and Wales by social and biological factors, 2011. Statistical Bulletin Published Online First: 20 November 2012.

4. National Collaborating Centre for Women's and Children's Health. Diabetes in pregnancy: management of diabetes and its complications from preconception to the postnatal period (Clinical Guideline). RCOG Press, 2008.

5. NICE. Dietary interventions and physical activity interventions for weight management before, during and after pregnancy. NICE public health guidance 27. National Institute for Health and Clinical Excellence, 2010.

6. NHS evidence (NICE). Clinical knowledge summaries: pre-conception-advice and managementmanagement. Which scenario? Secondary clinical knowledge summaries: pre-conception-advice and management-management. Which scenario?

http://www.cks.nhs.uk/pre_conception_advice_and_management/ management/which scenario

7. Heyes T, Long S, Mather's N. Preconception care: practice and beliefs of primary care workers. FAM Pract 2004; 21:22-7.

8. De-Regil L, Fernández-Gaxiola A, Dowswell T, et al. Effects and safety of periconceptional folate supplementation for preventing birth defects (review). Cochrane Database Syst Rev 2010;( 10):CD007950

9. Elsinga J, De Jong-Potjer LC, Van der Pal-de Bruin KM, et al. The effect of preconception counselling on lifestyle and other behavior before and during pregnancy. Women's Health Issues 2008; 18S: S117-25.

10. Lumley J, Donohue L. Aiming to increase birth weight: a randomized trial of pre-pregnancy information, advice and counselling in inner-urban Melbourne. BMC Public Health 2006; 6:299.

11. Howson C, Kinney M, Lawn J. Born too soon: the global action report on preterm birth. Geneva: WHO, 2012.

12. De Weerd S, Van der Bij AK, Cikot RJLM, et al. Preconception care: a screening tool for health assessment and risk detection. Prev Med 2002; 34:505-11. 
13. Jack B, Culpepper $L$, Babcock J, et al. addressing preconception risks identified at the time of a negative pregnancy test: a randomized trial. J FAM Pract 1998; 47:33-8.

14. Cefalo RC, Moos MK. Preconceptional health care: a practical guide. 2nd edn. St Louis, MO: Mosby, 1995.

15. Mazza D, Chapman A. Improving the uptake of preconception care and periconceptional folate supplementation: what do women think? BMC Public Health 2010; 10:786.

16. Hosli E, Elsinga J, Buitendijk S, et al. Women's motives for not participating in preconception counseling: qualitative study. Community Genet 2008; 11:166-70.

17. Helena Tuomainen, Laura Cross-Bardell, Mandeep Bhoday, Nadeem Qureshi, Joe Kai. Opportunities and challenges for enhancing preconception health in primary care: qualitative study with women from ethnically diverse communities. BCJ.2013

18. Canady RB, Tiedje LB, Lauber C. Preconception care and pregnancy planning: voices of African American women. MCN Am J Matern Child Nurs 2008; 33:90-7.

19. Chuang $\mathrm{CH}$, Vellot $\mathrm{DL}$, Weisman CS. Exploring knowledge and attitudes related to pregnancy and preconception health in women with chronic medical conditions. Matern Child Health J 2009; 14:713-19. Doi: 10.1007/s10995-009-0518-6 